\title{
Ethical issues related to clinical trials outside the International Conference on Harmonization regions
}

\author{
"There is a need for sustainable programs to develop local capacity to review and evaluate the ethics of \\ clinical trials outside The International Conference on Harmonization regions."
}

Keywords: clinical trials $\approx$ Declaration of Helsinki $=$ ethics guidelines $\approx$ ethical issues

The International Conference on Harmonization (ICH) of Good Clinical Practice forum was initiated in 1990 as a joint initiative involving both regulators and the pharmaceutical industry from Europe, the USA and Japan to discuss requirements for the scientific and technical aspects of product registration. The purpose was to maintain a forum to enable a dialogue between all parties and make recommendations towards greater harmonization.

During the 1990s, industry-sponsored research moved rapidly beyond Europe, the USA and Japan (the ICH regions) into large parts of Asia, Africa, Eastern Europe and Latin America. The expansion of clinical trials involved multiple stakeholders in a global environment where extreme wealth and poverty coexist. Globalization involves the conduct of clinical trials in developing countries, characterized by a large pool of patients, lower operating costs and with little or no regulatory constraints. Developing countries differ from $\mathrm{ICH}$ regions with regard to socioeconomic and cultural contexts. Cultural differences between $\mathrm{ICH}$ and non-ICH regions exist regarding moral values, traditions and community structures. Such differences have contributed to considerable controversies regarding the ethics of clinical trials that are sponsored by ICH regions and carried out in developing countries, raising issues about unfair practices in the distribution of benefits.

Over the past 50 years, several ethics guidelines for research involving human participants have been published and disseminated by international organizations and agencies such as the Declaration of Helsinki [101], Council for International Organizations of Medical Sciences [1] and UNESCO [2]. Ethical standards have been defined related to scientific design of the study, minimizing risks and maximizing benefits to create a favorable risk-benefit ratio, appropriate selection and recruitment of study participants, adequacy of medical care during the study and post-trial periods, compensation for any inconvenience and injury associated with the trial, protection of participant privacy and confidentiality, provision for proper informed consent and a prior review, and approval by ethics committees. Adherence to international guidelines by all parties involved in the clinical trial helps to promote ethical research and protect the rights and well-being of research participants and communities. However, the implementation of these guidelines in different contexts may not be as simple as one could imagine. A deep understanding of the principles laid out in the guidelines is required in order to apply them appropriately in the developing countries settings. In principle, the optimal protection of the participant must be a priority, no matter where a clinical trial is performed. However, replicating the same system of human participant protection in clinical trials from ICH regions to developing countries without considering their context may not ensure the same level of protection of research participants [102]. Many ethical controversies have ocurred when performing clinical trial outside the ICH regions, since the system of protection of participants in $\mathrm{ICH}$ region has been built up over decades based on different socioeconomic and cultural assumptions. The common issues are related to the informed consent process, research design specifically related to the use of placebo, post-trial benefits and ethics review.

\section{Informed consent}

The informed consent process that involves the way information is given to patients and the procedure of obtaining written consent may

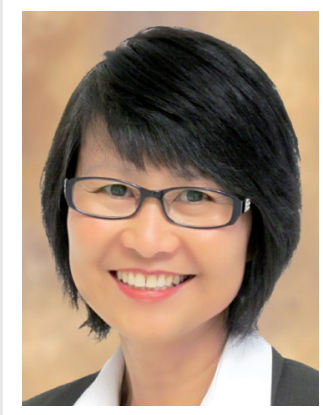

Juntra Karbwang

Author for correspondence: WHO, Avenue Appia 201211 Geneva 27, Switzerland Tel.: +41 227913867 Fax: +4I 227914774 E-mail: karbwangj@who.int

\section{Cristina Torres}

Forum for Ethical Review Committees in Asia and the Western Pacific, Clinical

Coordination and Training Center, Thammasat University,

Runsit Campus, Prathumthani

Province, Thailand 
vary according to specific country situations; for example, the level of literacy, understanding of the scientific issues and organization of the community. The situation may influence the consent procedures, particularly when vulnerable populations are recruited in a clinical trial. The involvement of people with knowledge of the local socioeconomic contexts, cultures and traditions that are able to protect the interests of those affected by the trial is necessary to guarantee the most appropriate informed consent procedures for potential participants in a clinical trial.

A large pool of naive patients available in developing country settings may facilitate quick recruitment, but at the same time raise ethical issues related to vulnerability and the corresponding level of protection required. Different socioeconomic and cultural contexts may require informed consent procedures different from the practices in the ICH regions. In resource-poor areas, where many patients are deprived of standard treatment, participation in a clinical trial provides an opportunity to have access to medical care. A hierarchical power relationship often exists between the doctor and the patient, and beneficence becomes of higher value than respecting the autonomy of the patient. Participants may also value the opinions of family members as important inputs to their decisions to participate in the trial and may want to formally involve them in the informed consent process. Such situations require more adroit procedures to address patient vulnerabilities and a higher level of cultural sensitivity to conduct an appropriate informed consent process. It requires putting the proper emphasis on relevant information in sections in the informed consent document and adjusting procedures to accommodate cultural differences.

\section{Research design}

When evaluating the efficacy of a new drug, the Declaration of Helsinki states that it should be compared with the best current proven intervention [101]. It also states clearly that a placebo may be used only when no current proven intervention exists or when there are compelling and scientifically sound methodological reasons, provided that the patients who receive the placebo will not be subject to any serious risk or irreversible harm. From the scientific methodological perspective, some conditions may require the use of placebo design to prove absolute efficacy of a new therapy; for example, new drugs for some chronic conditions with waxing and waning symptoms with high rate of placebo response [3]. The fundamental ethical principle underlying the application of this standard is the avoidance of exploitation, particularly for individuals or communities who may be vulnerable because of their socioeconomic status. Extreme care must be taken to avoid abuse of using a placebo option. There seems to be consensus on avoiding the use of a placebo in serious disease or harmful nonreversible conditions. When the best current intervention is not available locally, the best nationally available treatment or the so called 'established effective treatment' may be accepted, provided that it has acceptable side effects [1]. Research designs that compare new interventions with the best current proven intervention may be of little relevance in countries where such treatments are not available due to their high costs. It becomes important that local ethics committees critically review the justification for the selection of comparators within the context of their own health realities. It may not be realistic to apply the 'proven standard treatment' in locations where the existing infrastructures for healthcare services cannot support the best proven therapy for the local population. One example of using placebo control in a clinical trial in a developing country is the case of the Thailand trial to study a short-course zidovudine regimen to reduce mother-to-child transmission of HIV in 1997. The ethical justification for that study included the following [4]:

- There were significant differences in resources for healthcare infrastructures between the USA and Thailand, that is, the standard of perinatal care for the HIV-infected pregnant women in Thailand in 1997 did not include any HIV intervention at all. Then, the standard regimen for the prevention of HIV motherto-child transmission in the USA and Europe was not affordable and not implementable in most developing countries, where pregnant women made prenatal visits too late in their pregnancy to be able to receive a regimen that began early in the second trimester;

- The methodology superiority of the placebocontrolled trial was able to provide rapid and scientifically valid absolute efficacy results with fewer patients involved. The results of this study showed a $51 \%$ reduction in perinatal HIV transmission rates and the regimen became an established effective treatment in Thailand and other developing countries. 
When placebo control is used in a trial, not only is a careful assessment of a possible serious risk or irreversible harm essential, but the examination of a real need for the use of the placebo is required. The role of the ethics committee is pivotal in ensuring the implementation of this ethical guideline to avoid abuses related to placebo design. The ethics committee needs expertise to assess the justification of using placebo and to ensure that the participants will not be exposed to serious risk or irreversible harm.

\section{Risks \& post-trial benefits}

Issues related to access to care after the clinical trial is over are more complex in resource-poor regions where basic health insurance is not available. The Declaration of Helsinki states in article 14 that "the protocol should describe arrangement for post-study access by study subjects to intervention identified as beneficial in the study or access to other appropriate care or benefits". The Declaration deliberately requires the prior inclusion of this information and expects the ethics committee to make a judgment on the justification given in the protocol. Feasible posttrial access provisions need to be defined based on specific patient risks to maximize benefits that may be derived from participation. The outbreak of epidemics may facilitate quick enrolment in clinical trials but it may also raise ethical issues related to limiting the scope of access to successful products. Post-trial access is patient and country specific, and constitutes a major ethical hurdle that needs to be addressed by sponsors together with national regulatory authorities and ethics committees.

Socioeconomic differences between the sponsors in $\mathrm{ICH}$ regions and the host countries could affect the risk/benefit assessment of the research protocol. The risk/benefit ratio could be different from ICH countries since there is virtually no or limited health support infrastructure available for the population at large in developing countries. Participating in the trial may be the only means to have access to healthcare. In this case, the provision of basic healthcare becomes the additional responsibility of the sponsor towards the local community hosting a clinical trial and this responsibility may be extended even when the trial is over. It must be recognized that conducting clinical trials under international standards are more complicated in countries where health services are compromised. The researcher should exercise due diligence to maintain a favorable risk/benefit ratio and find innovative ways to address these issues. The local ethics committee should ensure that any semblance exploitation is avoided.

\section{The Ethics Review Committee}

The Declaration of Helsinki clarifies the role of ethics committees to review the protocol before the conduct of the study and to monitor an ongoing study. It is now widely accepted that the conduct of clinical trial can be initiated only after an appropriate ethics review and that the review should be done in countries where the clinical trial is to be conducted. It is expected that the local committees are familiar with the health needs, local health infrastructures, local culture and traditions, to be in the best position to render a balanced and reasoned judgment in reviewing a research protocol to arrive at a rational decision on what is appropriate for participants. Monitoring an ongoing study is one of the weaknesses of many ethics committees in developing countries due to the increasing number of protocols to review and the lack of resources to cope with their heavy workloads.

In 2001, the USA released a report entitled 'The Globalization of Clinical Trials: a Growing Challenge in Protecting Human Subjects [103]. The report marked a significant point in US policy toward the protection of human subjects in foreign clinical trials as it calls for a clear framework that will ensure the highest level of protection for participants of clinical trials in foreign countries. The report recommended that regulatory authorities and sponsors of foreign clinical trials should contribute to capacity building of foreign regulatory authorities with regard to the human subject protection, specifically the capacity of ethics committees that review biomedical research. Since then, a number of developing countries have received funding from US-established ethics review committees and have registered with the US Federal Wide Assurance System. Despite these initiatives, the proper functioning of ethics committees in foreign host countries remains to be a significant challenge. In 2006, the WHO Commission on Intellectual Property Rights, Innovation and Public Health acknowledged the need to raise the capacity for ethical review of research, stating that "although committees are being established, they are often of poor quality, because of limited training, and lack of awareness of international standards" [104].

The Forum for Ethical Review Committees in Asia and the Western Pacific has initiated a self-assessment and survey of ethical review 
practices in non-ICH regions since 2005 . The areas of weakness were identified related to international ethics principles and guidelines, and corresponding recommendations were given to address the weaknesses. The areas of weakness included incomplete compliance and noncompliance with their own standard operating procedures, inadequate membership to meet quorum requirements, inadequate review procedures, lack of monitoring mechanisms after approval, and inadequate documentation and archiving procedures. Recommendations for improvement include the conduct of relevant training and development of appropriate templates of procedures and forms for more efficient and effective review mechanisms. Such initiatives have raised the level of awareness and compliance with ICH standards with due consideration to local constraints and conditions among ethics committees, investigators and regulatory authorities.

Recognizing the implications of local socioeconomic and cultural conditions to the ethics of research, there is a need for sustainable programs to develop local capacity to review and evaluate the ethics of clinical trials outside ICH regions. Good ethical review is crucial to ensure that 'unethical trials' are not permitted in these countries. Non-ICH countries need to develop the proper legal and ethical frameworks, as well as develop the expertise of the ethics committees who review the protocols and associated documents. There is a need for concerted effort among research stakeholders in different geographical regions to address the threats and challenges to ethical research at the global arena.

\section{Financial \& competing interests disclosure}

The authors have no relevant affiliations or financial involvement with any organization or entity with a financial interest in or financial conflict with the subject matter or materials discussed in the manuscript. This includes employment, consultancies, honoraria, stock ownership or options, expert testimony, grants or patents received or pending, or royalties.

No writing assistance was utilized in the production of this manuscript.

\section{Bibliography}

1 Council for International Organizations of Medical Sciences/World Health Organization. International Ethical Guidelines for Biomedical Research Involving Human Subjects. WHO, Geneva, Switzerland (2002).

2 United Nations Educational, Scientific and Cultural Organization. Universal Declaration on Bioethics and Human Rights. United Nations Educational, Scientific and Cultural Organization, Paris, France (2006).

3 Temple R, Ellenberg SS. Placebo-controlled trials and active-control trials in the evaluation of new treatment. Part 1: ethical and scientific issues. Ann. Intern. Med. 133, 455-463 (2000).
4 Phanuphak P. Ethical issues in studies in Thailand of vertical transmission of HIV. $N$. Engl. J. Med. 338, 834-835 (1998).

\section{- Websites}

101 Declaration of Helsinki, 6th revision. www.wma.net/en/30publications/10policies/ b3/index.html (Accessed 5 June 2011)

102 The European Group on Ethics in Science and New Technologies to the European Commission. Opinion on the ethical aspects of clinical research in developing countries. www.eski.hu/new3/konyvtar/bookshop/ Opinion\%20No17.pdf

(Accessed 15 June 2011)
103 Department of Health and Human Services. The globalization of clinical trials: a growing challenge in protecting human subjects. www.who.int/intellectualproperty/ documents/thereport/ ENPublicHealthReport.pdf (Accessed 15 June 2011)

104 WHO. Public health innovation and intellectual property rights: report of the commission on Intellectual property rights, innovation and public health. www.who.int/intellectualproperty/ documents/thereport/ ENPublicHealthReport.pdf (Accessed 5 June 2011) 